eISSN 2444-7986

DOI: https://doi.org/10.14201/orl201782.15215

\author{
Artículo original
}

\title{
LOS PADRES COMO OBSERVADORES DE LA CALIDAD DE VIDA DE SUS HIJOS IMPLANTADOS COCLEARES
}

\section{The parents as assessors of the quality of life of their cochlear implanted children}

\author{
Silvia BORKOSKI-BARREIRO; Juan FALCÓN-GONZÁLEZ; Margarita TORRES-GARCÍA DE \\ CELIS; Isabel CHICHARRO-SORIA; Ángel RAMOS-MACÍAS.
}

Complejo Hospitalario Universitario Insular Materno Infantil de Gran Canaria. Servicio de Otorrinolaringología Cabeza y Cuello. Unidad de Hipoacusia. Las Palmas de Gran Canaria. España.

Correspondencia: silviaborkoski@hotmail.com

Fecha de recepción: 5 de noviembre de 2016

Fecha de aceptación: 30 de noviembre de 2016

Fecha de publicación: 2 de diciembre de 2016

Fecha de publicación del fascículo: 1 de junio de 2017

Conflicto de intereses: Los autores declaran no tener conflictos de intereses

Imágenes: Los autores declaran haber obtenido las imágenes con el permiso de los pacientes

Política de derechos y autoarchivo: se permite el autoarchivo de la versión post-print (SHERPA/RoMEO)

Licencia CC BY-NC-ND. Licencia Creative Commons Atribución-No comercial-Sin derivar 4.0 Internacional

๔ Universidad de Salamanca. Su comercialización está sujeta al permiso del editor 
Los padres son periodistas válidos sobre el estado de la calidad de vida global de sus hijos ya que dieron respuestas similares a las dadas por sus hijos.

PALABRAS CLAVE calidad de vida; implante coclear en niños

SUMMARY

Introduction and objective: Quality of life refers to the hability of an individual to enjoy normal life activities, including aspects of functional capacity and well-being. The concept of quality of life has been increasingly used in the field of health assessments. Only recently have the effects of Cochlear implantation from the perspective of emotional and social development begun to be investigate. To examine the results of health-related quality of life in cochlear implanted children between 11 and 12 years of age and their parents. Method: Observational, transversal descriptive study .24 unilateral or successive bilateral children implanted prelingually between 11 and 12 years of age and their parents. The pediatric quality of life questionnaire Persil ${ }^{\mathrm{TM}}$ (Version 4.0, Spanish for Spain) was used to measure health-related quality of life. It was completed independently by children and parents. Results: The results were analyzed by means of a T -Student test. The outcomes of children and parents at different scales evaluated show that statistically significant results appear on scales of emotional and academic functioning $(p<0.05)$. When comparing the overall result of the questionnaire with the different scales evaluated, all show statistically significant results $(p<0.05)$. Discussion: It has been proven the success of a cochlear implant in speech and language field, this leads us calls into question beyond these areas; yield at home, at school and in different social contexts. There are few studies that explain through parental questionnaires expectations and their perception of the quality of life in cochlear implanted in children. Conclusions: The results of the study show that children enjoy the benefits of using a cochlear implant. They report benefits in all areas studied (physical, emotional, social and academic). Parents are reliable reporters about the state of the overall quality of life of their children since their answers were similar to those given by their children.

quality of life; cochlear implant in children

\section{INTRODUCCIÓN}

Los problemas auditivos deben ser reconocidos tan tempranamente en la vida del niño como sea posible, para que el proceso de habilitación auditiva aproveche la plasticidad del sistema sensorial en desarrollo y así evitar la consecuencia más importante de la hipoacusia infantil: crecer sin lenguaje, comprometiendo su futura integración social y laboral.

Está aceptada universalmente la utilidad y eficacia de la implantación coclear en niños con diagnóstico de hipoacusia neurosensorial profunda. El progreso se ha observado en el desarrollo del lenguaje, pero se ha empezado a investigar los efectos de la implantación a nivel del desarrollo social y emocional del niño [1-7]. El concepto de calidad de vida ha comenzado a utilizarse cada vez más en el campo de las evaluaciones en salud o como medida de bienestar.

La OMS (1994) define la "calidad de vida" como la percepción del individuo sobre su posición en la vida dentro del contexto cultural y el sistema de valores en el que vive y con respecto a sus metas, expectativas, normas y preocupaciones. Se refiere a la calidad del individuo para disfrutar de las actividades normales de la vida, incluyendo conceptos de capacidad funcional y bienestar [8].
Pocos estudios han observado directamente 0 cuestionado acerca de la calidad de vida de los niños con implantes cocleares y la percepción de la misma de sus padres [9-11].

Algunos estudios observan que un importante porcentaje de los niños sordos están preocupados con la falta de amistad y aceptación social o tienen dificultades para la interacción o aceptación social, en comparación con niños con audición normal $[12,13]$.

La transición desde la infancia a la preadolescencia y adolescencia a menudo se complica y aún más cuando un niño tiene una pérdida auditiva asociada, con el fin de estudiar el impacto del IC en los que se encuentran en esta etapa y la visión de sus padres se ha llevado a cabo este estudio utilizando el cuestionario de calidad de vida pediátrica PedsQL ${ }^{\mathrm{TM}}$, uno de los cuestionarios de calidad vida pediátrico más utilizado $[14,15]$.

\section{MATERIAL Y MÉTODO}

Estudio observacional, descriptivo transversal en 24 sujetos con diagnóstico de hipoacusia prelocutiva, portadores de implante coclear (IC) unilateral o bilateral, de 11 y 12 años de edad implantados entre los 1 y 5 años. El período interimplante en la modalidad bilateral sucesivo es menor e igual a 7 años. Todos con 
inserción completa del haz de electrodos implantados en nuestro Servicio.

Se utilizó el cuestionario de calidad de vida pediátrico «PedsQL ${ }^{\mathrm{TM}}$ » [15] (Versión 4.0 - español para España) a ser completado por los padres y niños el mismo día; valorando de forma independiente la percepción de calidad de vida de los niños por ellos mismos y sus padres. El cuestionario PedsQL ${ }^{\mathrm{TM}}$ consta de cuatro escalas: funcionamiento físico, funcionamiento emocional, funcionamiento social y de desempeño escolar que mide la calidad de vida en relación a las cuatro últimas semanas.

Las repuestas se puntúan del 0 al 4 , donde 0 es nunca, 1 casi nunca, 2 a veces, 3 a menudo y 4 siempre, los elementos se pueden transformar linealmente a una escala de $0-100$, de la siguiente manera: $0=100 ; 1=75 ; 2=50 ; 3=$ 25 y $4=0$. Siendo los valores máximos de CVRS (calidad de vida relacionada con la salud) a obtener en las escalas emocionales, funcionamiento social y la escuela de 500 y en la escala de salud psicosocial de 800.

Las puntuaciones más altas indican una mejor CVRS. Las puntuaciones de la escala no deben ser calculadas si los elementos de la misma no son respondidos en más del $50 \%$.

Para el resultado en relación a la salud psicosocial, la media se calcula como la suma de los elementos más el número de ítems contestados en las escalas emocionales, funcionamiento social, y la escuela.

La puntuación de salud física es la misma que la puntuación de la escala funcionamiento físico. Para el resultado total, la media se calcula como la suma de todos los elementos sobre el número de ítems contestados en todas las escalas.

Para el procesamiento estadístico de los datos se utilizó el SPSS (versión 20.0).El contraste de hipótesis se consideró estadísticamente significativo cuando el correspondiente $p$-valor fue inferior a 0.05. La comparación estadística para muestras independientes se realizó utilizando la prueba de T-Student.

El Estudio obtuvo la aprobación del comité ético de nuestro complejo hospitalario.

\section{RESULTADOS}

Análisis descriptivo. Se administró el cuestionario a 24 sujetos implantados, de entre $11 \mathrm{y}$ de 12 años de edad; 14 niñas (58 \%) y 10 niños $(42 \%)$ y 24 padres.

En el total de la muestra la media del resultado total es del 80,54 (rango 45,5-97,7), en cuanto a las medias obtenidas en las diferentes escalas se observa que en la escala de funcionamiento físico es de 666,31 (rango 283-808); en el funcionamiento emocional es de 406,10 (rango 130-505), en el funcionamiento social es de 416,46 (rango 155-505) y en el funcionamiento Escolar es de 362,19 (rango 205-505).

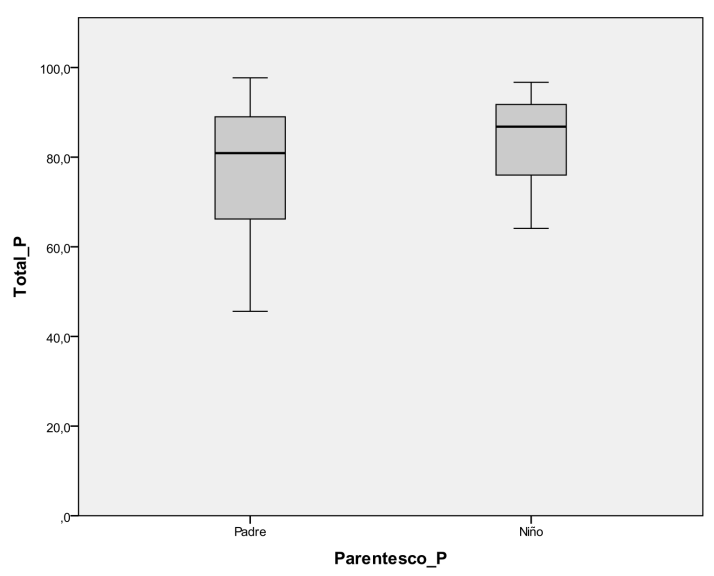

Figura 1. Media del resultado total del cuestionario (padres - niños).

En la muestra de manera individual la media del resultado total del cuestionario es de 77,42 para el grupo de los padres y de 83,65 para el de los niños (Figura 1).

Tabla 1. Medias obtenidas en las diferentes dimensiones evaluadas.

\begin{tabular}{|c|l|c|c|c|c|}
\cline { 2 - 6 } \multicolumn{1}{c|}{} & \multicolumn{1}{|c|}{ Escalas } & Mínimo & Máximo & Media & $\mathrm{dt}$ \\
\hline $\begin{array}{c}\text { Padres } \\
\mathrm{n}=24\end{array}$ & Función Física & 283 & 808 & 662,04 & 159,01 \\
\cline { 2 - 6 } & $\begin{array}{l}\text { Función } \\
\text { Emocional }\end{array}$ & 130 & 505 & 378,04 & 100,02 \\
\cline { 2 - 6 } & Función Social & 155 & 505 & 400,83 & 102,86 \\
\cline { 2 - 6 } & $\begin{array}{l}\text { Desempeño } \\
\text { Escolar }\end{array}$ & 205 & 505 & 337,29 & 81,59 \\
\cline { 2 - 6 } & $\mathrm{n}=24$ & 45,6 & 97,7 & 77,421 & 15,04 \\
\cline { 2 - 6 } & $\begin{array}{l}\text { Función Total } \\
\text { Emocional }\end{array}$ & 280 & 505 & 434,17 & 67,02 \\
\cline { 2 - 6 } & Función Social & 305 & 505 & 432,08 & 62,95 \\
\cline { 2 - 6 } & $\begin{array}{l}\text { Desempeño } \\
\text { Escolar }\end{array}$ & 205 & 505 & 387,08 & 76,95 \\
\cline { 2 - 6 } & & 64,1 & 96,7 & 83,654 & 10,25 \\
\hline
\end{tabular}

Las medias obtenidas en las diferentes dimensiones evaluadas y en los grupos de niños y 
padres de manera individual se describen en la tabla 1 y figuras $2,3,4$ y 5 .

Al analizar mediante la T-Student los resultados de niños y padres en las diferentes escalas evaluadas observamos que en las escalas de funcionamiento emocional y académica se observan diferencias entre lo que responden los padres y los niños siendo éstos resultados estadísticamente significativos $(p<0,05)$.

Mientras que, al analizar mediante T-Student para muestras relacionadas observamos que, al comparar el resultado total del cuestionario con las diferentes escalas evaluadas, en todas ellas se obtienen resultados estadísticamente significativos $-p<0,001)-($ Tabla 2$)$.

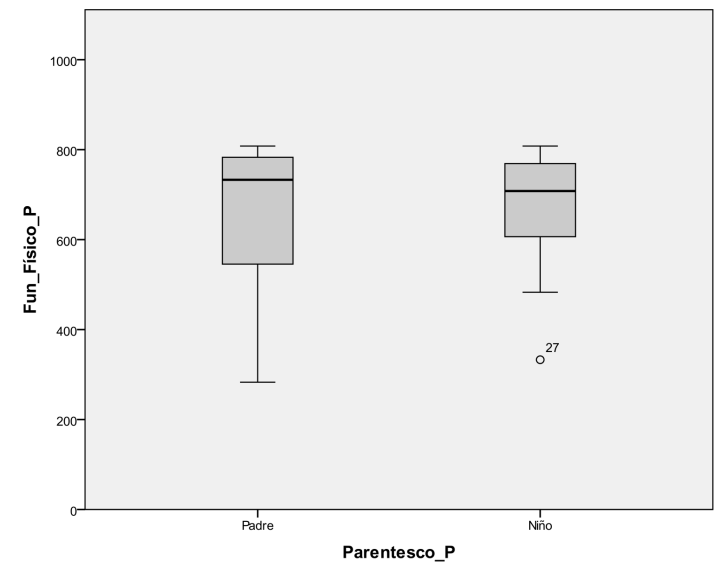

Figura 2. Medias obtenidas en la dimensión Función Física.

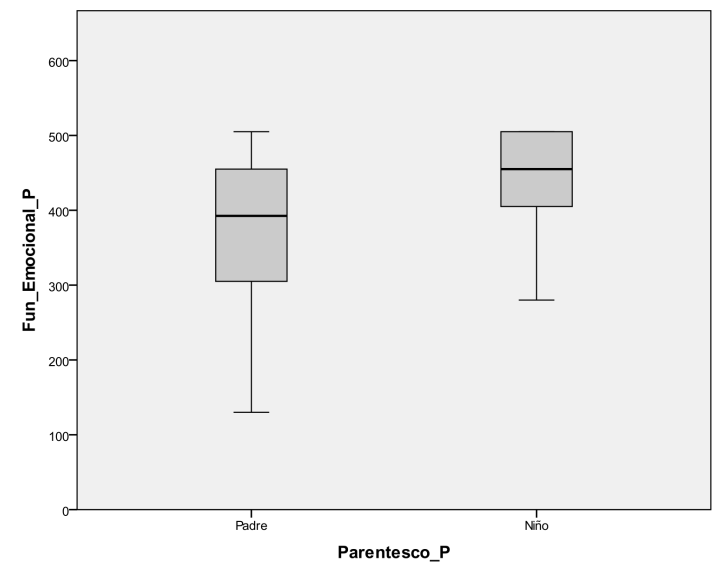

Figura 3. Medias obtenidas en la dimensión función emocional.

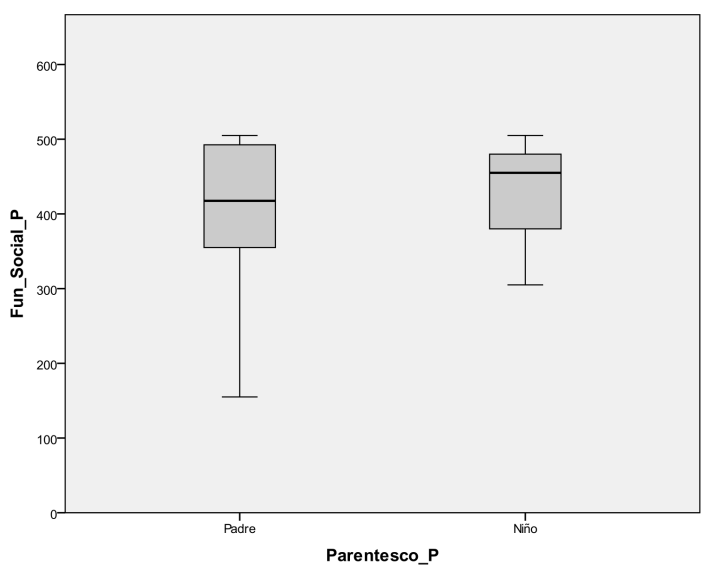

Figura 4. Medias obtenidas en la dimensión función social.

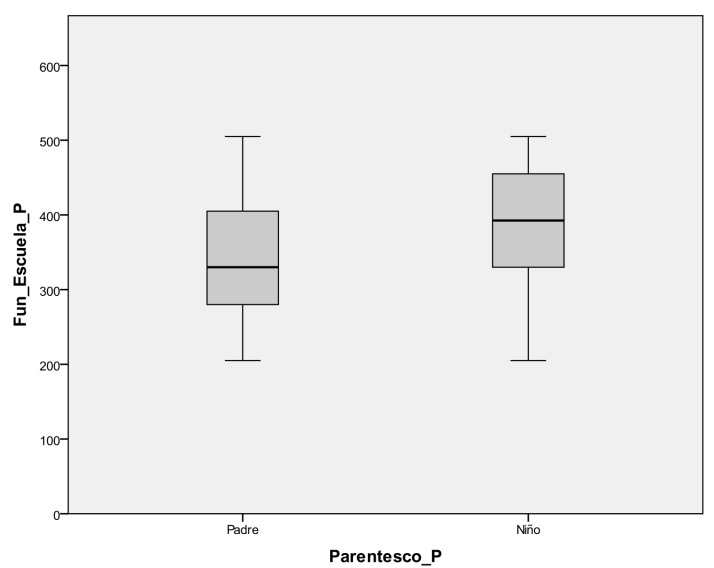

Figura 5. Medias obtenidas en la dimensión función escuela.

\section{DISCUSIÓN}

Estudios de los últimos años han demostrado la eficacia del IC como tratamiento para niños con diagnóstico de hipoacusia severo-profunda, mejorando las habilidades de la comunicación.

Los estudios sobre el impacto de IC se han centrado en la evaluación en sus medidas de eficacia clínica (habilidades auditivas, lenguaje, umbrales de audición). Estas medidas constituyen sólo una pequeña parte del efecto que un IC tiene en la vida de un niño [16].

Probado el éxito del IC en las áreas del habla y del lenguaje, esto nos lleva actualmente a preguntas más allá de estas áreas; el rendimiento en el hogar, en la escuela y en diferentes contextos sociales [17]. 
Tabla 2. Comparación del resultado total del cuestionario con las diferentes escalas evaluadas.

\begin{tabular}{|c|c|c|c|c|c|}
\hline Escalas & Media & $\mathrm{dt}$ & Inferior & Superior & $\mathrm{p}^{*}$ \\
\hline $\begin{array}{l}\text { Función } \\
\text { física }\end{array}$ & 585.78 & 132,63 & 547,26 & 624,29 & \multirow{4}{*}{$<0.001$} \\
\hline $\begin{array}{l}\text { Función } \\
\text { emocional }\end{array}$ & 325,57 & 78,74 & 302,70 & 348,44 & \\
\hline $\begin{array}{l}\text { Función } \\
\text { social }\end{array}$ & 335,93 & 75,90 & 313,88 & 357,96 & \\
\hline $\begin{array}{l}\text { Desempeño } \\
\text { escolar }\end{array}$ & 281,65 & 74,95 & 259,89 & 303,41 & \\
\hline
\end{tabular}

Diferentes autores afirman que los niños sordos se sienten menos aceptados socialmente y tienen más dificultades para hacer amigos y son significativamente más impulsivos que sus pares oyentes $[13,18,19]$.

Pocos estudios exploran a través de entrevistas o cuestionarios las expectativas de los padres, su satisfacción con la implantación coclear, aspectos estresantes de esta proceso y la percepción de la calidad de vida de sus hijos usuarios de implantes coclear $[10,11,20$, 21].En una revisión sistemática de los estudios de calidad de vida relacionada con la salud que afectan a niños implantados cocleares, Lin y Niparko encontraron sólo diez estudios en los que los participantes tenían menos de 18 años y donde se utilizó un cuestionario en inglés con dominios psicosociales de la salud física, mental, y social [17].

Existe un solo estudio en lengua española donde se busca conocer los beneficios en la calidad de vida en pacientes adultos y pediátricos portadores de IC [22].

$\mathrm{Al}$ analizar las escalas de manera individual y en ambos grupos observamos que las medias obtenidas por los niños son mayores, aunque similares a las referidas por sus padres, siendo este hallazgo importante en sí mismo ya que nos refleja la satisfacción por parte de los niños usuarios de IC. De acuerdo con la literatura, nuestros resultados muestran el efecto positivo del IC en todos los aspectos [16-23].

Loy et al. demostraron que los niños con sordera profunda que utilizaban de manera regular su IC, los sentimientos sobre la vida en general no eran mejores ni peores que sus compañeros oyentes. En consonancia los niños del estudio de Chmiel et al., al responder un cuestionario de 5 preguntas directas reportaron, mejoras significativas en su calidad de vida. Ambos autores cuando compararon las respuestas de los hijos con las de sus padres observaron que dieron respuestas similares. Resultados semejantes a los obtenidos en nuestro trabajo, donde observamos que los padres son observadores fiables en relación a la percepción de la calidad de vida global de sus hijos, ya que coinciden con las propias opiniones de los niños sobre el estado de su salud en el momento valorado $[20,24]$.

\section{CONCLUSIONES}

Los niños en este estudio en el momento en que se analiza su calidad de vida, disfrutan de los beneficios del uso de su IC. Reportan beneficios en todas las áreas estudiadas (física, emocional, social y académica). Creemos que son necesarios más trabajos con muestras mayores que investiguen la calidad de vida relacionada con la salud en la población pediátrica de lengua española portadores de IC, utilizando instrumentos validados para tal fin, para observar las experiencias únicas de estos niños y los problemas del desarrollo a medida que maduran y llegan a la adolescencia.

Los padres mostraron resultados similares a los de sus hijos demostrando poder ser buenos al evaluar la calidad de vida global de sus hijos.

\section{BIBLIOGRAFÍA}

1. Kirk KI, Miyamoto RT, Lento $\mathrm{CL}$, Ying $\mathrm{E}$, O'Neill T, Fears B. Effects of age at implantation in young children. Ann Otol Rhinol Laryngol. 2002;111:69-73.

2. Svirsky, M. A., Teoh, S., Neuberger, H. Development of language and speech perception in congenitally, profoundly deaf children as a function of age at cochlear implantation. Audiol Neurootol. 2004;9:22433.

3. Houston D, Pisoni D, Kirk K, Yin E, Miyamoto R. Speech perception skills of deaf infants following cochlear implantation. A first report. Int J Pediatr Otorhinolaryngol. 2003;67:47995.

4. Al-Muhaimeed $\mathrm{H}$. Assessment of auditory performance in young children with cochlear implants. Cochlear Implants Int. 2010; 11(Suppl 1):240-3.

5. Robbins AM, Osberger AJ, Miyamoto RT, Kessler KS. Language development in young children with cochlear implants. Adv Otorhinolaryngol. 1995;50:160-6. 
6. Manrique M, Cervera-Paz FJ, Huarte A, Molina M. Advantages of cochlear implantation in prelingual deaf children before 2 years of age when compared with later implantation. 2004;114(8):1462-9.

7. Gordon KA, Papsin BC. Benefits of short interimplant delays in children receiving bilateral cochlear implants. Otol Neurotol, 2009;30:319-331.

8. Guyatt $\mathrm{GH}$, Feeney DH Patrick, DL. Measuring health related quality of life. Ann Intern Med. 1993; 15;118(8):622-9.

9. Thoutenhoofd E, Archbold S, Gregory S, Lutman ME, Nikolopoulos TP, Sach T. Paediatric cochlear implantation: evaluating outcomes. Elsevier; 2005.

10. Marschark M. Origins and interactions in the social, cognitive, and language development of deaf children. In: Marschark M, Clark D (Eds.). Psychological Perspectiveson Deafness, Lawrence Erlbaum Associates. New Jersey: Hillsdale; 1993.

11. Spilker B, Revicki D. Taxonomy of Quality of Life. In: Quality of Life and Pharmaco economics in Clinical Trials. Spilker B (Ed.). Baltimore: Lippincott Williams \& Wilkins; 1996.

12. Davis JM, Elfenbein J, Schum R, Bentler RA. Effects of mild and moderate hearing impairments on language, educational, and psychosocial behavior of children. J Speech Hear Disord 1986;51:53-62.

13. Knutson JF, Boyd RC, Reid JB, Mayne T, Fetrow R. Observational assessments of the interaction of implant recipients with family and peers: Preliminary findings. Otolaryngol Head Neck Surg 1997;117:196-207.

14. Upton P, Lawford J, Eiser C. Parent-child agreement across child health-related quality of life instruments: a review of the literature. Qual Life Res 2008; 17: 895-913.
15. The PedsQL ${ }^{\mathrm{TM}}$ Measurement Model for the Pediatric Quality of life Inventory ${ }^{\mathrm{TM}}$. James W. Varni. En: http://www.pedsql.org

16. Fortunato-Tavares T, Befi-Lopes D, Ferreira Bento R, Furquim de Andrade CR. Children with cochlear implants: communication skills and quality of life. Braz J Otorhinolaryngol. 2011;78(1):15-25.

17. Lin F, Niparko J. Measuring health-related quality of life afterpediatric cochlear implantation: A systematic review. Int J Pediatr Otorhinolaryngol. 2006; 70: 16951706.

18. Cappelli $M$, Daniels $T$, Durieux-Smith $A$. Social development of children with hearing impairments who are integrated into general education classrooms. Volta Review 1995;97:197-208.

19. Loy B, Andrea D. Warner-Czyz, Liyue Tong, Emily A. Tobey, Peter S. Roland, The children speak: An examination of the quality of life ofpediatric cochlear implant users. Otolaryngol Head Neck Surg. 2010; 142(2): 247-53.

20. Vidas S, Hassan R, Parnes LS. Real-life performance considerations of four pediatric multi-channel cochlear implant recipients. J Otolaryngol. 1992;21(6):387-93.

21. Spilker B, Revicki D. Taxonomy of Quality of Life. In: Quality of Life and Pharmaco economics in Clinical Trials. Spilker B (Ed.). Baltimore: Lippincott Williams \& Wilkins; 1996.

22. Castro A, Lassaletta L, Bastarrica M, Alfonso M, Prim MP, de Sarriá MJ, Gavilán J. Calidad de vida en pacientes con implante coclear. Acta Otorrinolaringol Esp 2005;55:192-7.

23. Kluwin TN, Stewart DA. Cochlear implants for younger children: a preliminary description of the parental decision process and outcomes. Am Ann Deaf. 2000;145(1):26-32.

24. Chmiel, R., Sutton, L., Jenkins, H. Quality of life in children with cochlear implants. Annals of Otology,Rhinology and Laryngology Supplement.2000;185:100-3. 